\title{
Prediction of type 1 diabetes among siblings of affected children and in the general population
}

\author{
H. T. Siljander $\cdot$ R. Veijola $\cdot$ A. Reunanen . \\ S. M. Virtanen • H. K. Åkerblom • M. Knip
}

Received: 21 March 2007 / Accepted: 9 July 2007 / Published online: 4 September 2007

(C) Springer-Verlag 2007

\begin{abstract}
Aims/hypothesis To compare the predictive characteristics of autoantibodies to GAD (GADA) and islet antigen 2 (IA2A) for type 1 diabetes between siblings of affected children and children from the general population.

Methods Seven-hundred and fifty-five siblings and 3,475 population-derived children were screened for GADA and IA-2A and observed for type 1 diabetes for 15 years. Sensitivity and cumulative disease risks from GADA, IA$2 \mathrm{~A}$ and double positivity were compared between the cohorts.

Results Fifty-six siblings (7.4\%) tested positive for GADA, $39(5.2 \%)$ for IA-2A and 29 (3.8\%) for both autoantibodies. Thirty-four population derived participants $(1.0 \%)$ had
\end{abstract}

\footnotetext{
H. T. Siljander $\cdot$ H. K. Åkerblom • M. Knip ( $₫)$

Hospital for Children and Adolescents, University of Helsinki, P.O. Box 22 (Stenbäckinkatu 11), 00014 Helsinki, Finland e-mail: mikael.knip@hus.fi
}

H. T. Siljander $\cdot$ M. Knip

Department of Paediatrics, Tampere University Hospital,

Tampere, Finland

\section{R. Veijola}

Department of Paediatrics, University of Oulu,

Oulu, Finland

A. Reunanen $\cdot$ S. M. Virtanen

National Public Health Institute,

Helsinki, Finland

S. M. Virtanen

Tampere School of Public Health, University of Tampere,

Tampere, Finland

S. M. Virtanen

Research Unit, Tampere University Hospital,

Tampere, Finland
GADA, $22(0.6 \%)$ had IA-2A and $7(0.2 \%)$ had double positivity. Fifty-one siblings $(6.8 \%)$ and 15 participants in the population cohort $(0.4 \%)$ progressed to type 1 diabetes. The predictive sensitivity of GADA was $68 \%$ (95\% CI $53-$ $81 \%)$ among siblings and $50 \%(95 \%$ CI $23-77 \%)$ in the general population, while the corresponding values were 58 (95\% CI $43-72 \%)$ and $43 \%$ (95\% CI $18-71 \%$ ) for IA-2A. Double-autoantibody positivity had a sensitivity of $48 \%$ (95\% CI 34-63\%) among siblings and 36\% (95\% CI $13-$ $65 \%$ ) in the population cohort. Cumulative disease risks from GADA, IA-2A and double positivity were, respectively, $61 \%$ (95\% CI $48-74 \%$ ), $74 \%$ (95\% CI 61-88\%) and 83\% (95\% CI 69-97\%) among siblings compared with those of $24 \%$ (95\% CI 9-38\%), 32\% (95\% CI $12-51 \%$ ) and $86 \%$ (95\% CI 60-100\%) in the general population.

Conclusions/interpretation There were no significant differences in the disease-predictive sensitivity of GADA and IA-2A positivity or their combination between siblings and the population cohort, whereas, for each antibody, positivity was associated with a higher cumulative disease risk among siblings. Double-antibody positivity conferred similar cumulative disease risk both among siblings and in the general population.

Keywords Autoantibodies - General population · Prediction · Siblings · Type 1 diabetes
Abbreviations
DiMe The Childhood Diabetes in Finland Study
GADA autoantibodies against GAD
IAA autoantibodies against insulin
IA-2A autoantibodies against islet antigen 2
ICA islet cell antibodies
LASERI The Study on Cardiovascular Risk Factors in Young Finns 


\section{Introduction}

The first data on the predictive value of autoantibodies for type 1 diabetes were obtained by screening first-degree relatives of affected patients for islet cell antibodies (ICA), which represent a heterogeneous group of antibodies to a series of known islet antigens, including autoantibodies against islet antigen 2 (IA-2A) and GAD (GADA), as well as antibodies to one or more so far unidentified antigens [1]. More recent studies have indicated that while identifying first-degree relatives with an elevated disease risk, ICA screening could be replaced with screening for GADA and IA-2A [2], and accordingly current guidelines for the primary testing of this population recommend the measurement of GADA along with either IA-2A or autoantibodies against insulin (IAA) [3]. However, no guidelines for the identification of at-risk individuals from the general population have so far been published, although approximately $90 \%$ of new cases with type 1 diabetes arise from that population. Although information on the predictive characteristics of diabetes-associated autoantibodies in the general population is limited, there are indications that the predictive value of positivity for multiple autoantibodies, i.e. two or more, is close to that observed among first-degree relatives $[4,5]$. We present here direct comparisons of the predictive characteristics of GADA, IA-2A and their combination between first-degree relatives and a representative general population cohort over an observation period of 15 years.

\section{Methods}

The current prospective observational study employed two cohorts of healthy Finnish children and adolescents, derived from the Childhood Diabetes in Finland (DiMe) study [6] and the Study on Cardiovascular Risk Factors in Young Finns (LASERI) [7]. The 755 DiMe study participants (348 male) were non-diabetic siblings of children with newly diagnosed type 1 diabetes recruited between 1986 and 1989 and observed until the end of 2002. The 3,475 participants (1,714 male) in the LASERI study were randomly selected 3 - to 18-year-old individuals from the general population in the five university hospital referral areas in Finland. The study was initiated in 1980, and the follow-up for this report lasted until the end of June 1995. The mean age at the first blood sampling was 10.0 years (range 1.3-19.9 years) for DiMe siblings and 10.8 years (range $2.7-18.9$ years) in the LASERI general population cohort. The median follow-up time was 15 years in both groups (range 13.7-16.3 years among siblings and 14.8-15.1 years in the general population cohort). Participants were observed for progression to overt type 1 diabetes, the criteria of which were in accordance with the WHO definitions [8]. Cases from both cohorts were ascertained from the Central Drug Registry of the National Social Insurance Institute, which has an ascertainment rate of more than $99 \%$ for new patients with type 1 diabetes in Finland [9]. The protocols of the DiMe and LASERI studies had been approved by the local ethics committees and informed consents were attained before the commencement of the study.

Procedures This survey was based on a single blood sample for each participant, obtained as soon as possible after the diagnosis of the index case in the sibling cohort, and at the first visit to a study centre in the general population cohort. Samples were analysed between 2002 and 2004 for GADA and IA-2A with specific radiobinding assays [10], the cutoff limits for positive results (99th percentiles of more than 370 Finnish non-diabetic children) being $14.1 \mathrm{WHO}$ units $/ \mathrm{ml}$ (5.36 relative units) for GADA and $1.9 \mathrm{WHO}$ units $/ \mathrm{ml}(0.43$ relative units) for IA-2A. The disease sensitivity of the GADA assay was $82 \%$ and the specificity $97 \%$ in the 2003 Diabetes Autoantibody Standardisation Programme Workshop, while the corresponding characteristics for the IA-2A assay were 64 and $100 \%$. All samples with autoantibody levels between the 97th and 99.5th percentiles were reanalysed to confirm their status.

Data analysis Sensitivities of GADA, IA-2A and combined positivity were estimated in the two study cohorts based on the minimum follow-up time of 13.7 years. The cumulative disease risks were assessed based on the median follow-up time of 15 years; $95 \%$ CIs were assessed by the exact method and distributions tested with the $\chi^{2}$ test. The Kaplan-Meier method with the log-rank test was used to construct life tables for the likelihood of remaining diabetes-free and to compare the disease-free survival rate distributions. A twotailed $p$ value of 0.05 was considered to indicate statistical significance. SPSS (Version 11.0; SPSS, Chicago, IL, USA) was used for the statistical analyses.

\section{Results}

Fifty-six siblings (7.4\%) tested positive for GADA and $39(5.2 \%)$ for IA-2A. Positivity for either of the autoantibodies was observed in 66 siblings $(8.7 \%)$ and double positivity in 29 siblings $(3.8 \%)$. In the general population cohort, 34 participants $(1.0 \%)$ were positive for GADA, $22(0.6 \%)$ for IA-2A and $49(1.4 \%)$ for one or the other. Seven children $(0.2 \%)$ tested positive for both autoantibodies.

Fifty-one siblings $(6.8 \% ; 25$ male) and 15 participants $(0.4 \%$; ten male $)$ in the general population cohort progressed to type 1 diabetes during the observation period. The mean 


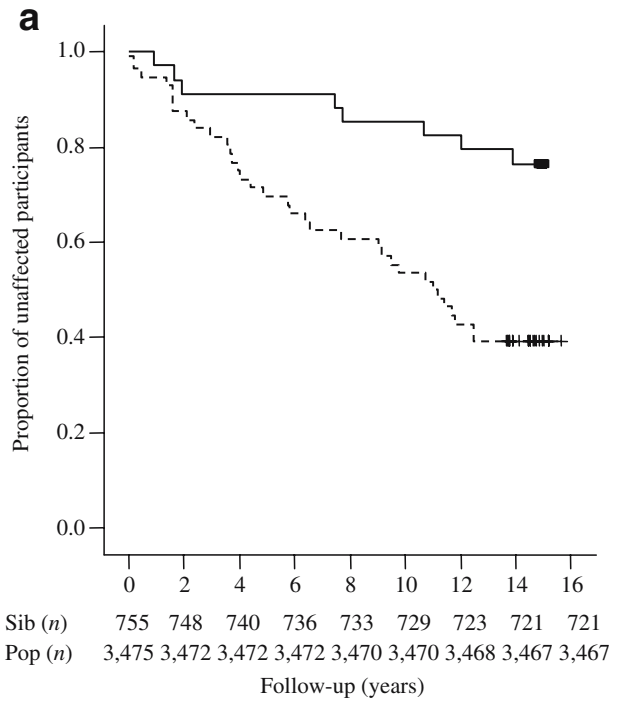

b

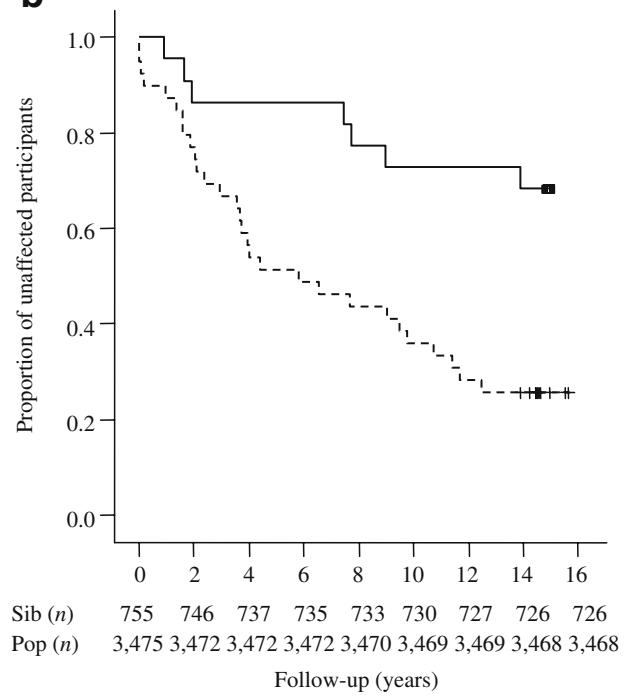

C

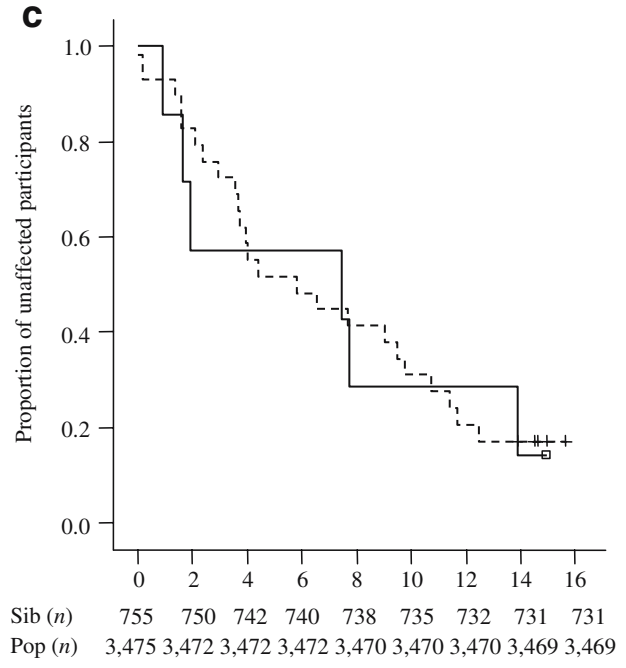

Follow-up (years)

Fig. 1 Kaplan-Meier diabetes-free survival rate analyses of the siblings (broken lines) and population cohorts (solid lines) in relation to positivity for GADA $(\mathbf{a} ; p=0.0007)$, IA-2A $(\mathbf{b} ; p=0.0017)$ or both autoantibodies $(\mathbf{c} ; p=0.89)$. Sib, siblings; Pop, general population age at the diagnosis was 14.1 years (range $1.5-28.4$ years) in siblings and 16.8 years (range 5.5-32.8 years) in the population cohort. The mean interval from the blood sampling to diagnosis was 5.9 years (range $0.01-13.7$ years) in siblings and 7.3 years (range $0.9-3.9$ years) in the population cohort.

The predictive sensitivity of GADA positivity was $68 \%$ (95\% CI 53-81\%) among siblings and 50\% (95\% CI $23-$ $77 \%$ ) in the general population, while the corresponding figures were $58 \%$ (95\% CI $43-72 \%)$ and $43 \%$ (95\% CI $18-$ $71 \%$ ) for IA-2A positivity. Combined positivity for GADA and IA-2A had a sensitivity of $48 \%$ (95\% CI $34-63 \%)$ among siblings, the corresponding value being $36 \%(95 \%$ CI $13-65 \%$ ) in the population cohort. Accordingly, positivity for GADA conferred the highest predictive sensitivity in both cohorts, but none of the sensitivity estimates differed significantly between the two groups.

Analyses of the diabetes-free survival rates of GADA-, IA-2A- and double-positive children are presented in Fig. 1. Cumulative disease risk from GADA positivity was higher in siblings $(61 \%$; $95 \%$ CI $48-74 \%)$ than in participants from the population cohort (24\%; 95\% CI 9$38 \% ; p=0.0007)$. Similarly, IA-2A positivity was associated with a higher progression rate among siblings $(74 \%$; $95 \%$ CI $61-88 \%)$ than in the general population (32\%; 95\% CI $12-51 \% ; p=0.0017$ ), while double positivity carried similar cumulative risk in both populations, i.e. $83 \%(95 \%$ CI 69-97\%) among siblings and 86\% (95\% CI 60-100\%; $p$ $=0.89$ ) in the general population.

\section{Discussion}

This study represents a direct comparison of the predictive characteristics of diabetes-associated autoantibodies between first-degree relatives and a population-based cohort. The virtues of this work lie in its use of a longer observation period than in any previous survey, the comparable age range at the start of observation in both series and the relatively extensive population cohort, recruited in the country with the highest incidence of type 1 diabetes in the world [9].

In our study, more than $80 \%$ of the participants testing positive for both GADA and IA-2A developed type 1 diabetes during a 15 year observation period and the proportion of progressors was similar among siblings of affected patients and in the general population. However, since the single-point, double positivity identified only $40-50 \%$ of the progressors, repeated testing for all four predictive autoantibodies (ICA, IAA, GADA and IA-A2) would most likely have increased the sensitivity of the screening procedure. HLA genotyping of the general population cohort would have identified a group of individuals at increased diabetes risk for subsequent monitoring of diabetes-associated autoantibodies. Unfortunately, no such data were compre- 
hensively available for both study cohorts, and therefore a detailed analysis of autoantibody combinations and their relationships with genetic risk markers could not be performed.

Each of the present two markers as well as their combination tended to have somewhat higher sensitivity among siblings than in the population cohort, but the differences did not reach statistical significance. Positivity for multiple autoantibodies is a rare phenomenon in the general population, and since only seven individuals in the population cohort developed double positivity, the statistical power to confirm the similar progression rates in the two cohorts remained relatively weak. However, from previous studies we know that an overwhelming majority of the progressors develop multiple-autoantibody positivity months to years before the diagnosis, and that combined GADA and IA-2A screening identified all single positive and $96 \%$ of the multiple-positive progressors among the DiMe siblings and all European and American schoolchildren testing positive for multiple antibodies [4-6]. Our data confirmed the previously published observation that a single-point negative autoantibody test is associated with an extremely low risk of developing type 1 diabetes, in the general population in particular [11].

Any population screening programme started at a mean age of 10 years can be estimated to miss a notable proportion of the progressors, since the cumulative incidence of type 1 diabetes by the age of 10 years is approximately $40 \%$ of that recorded by the age of 30 years [12]. Therefore, if the target of the screening programme is to maximise the number of future patients covered by the programme, screening has to be initiated early in life. This will become an even more important aspect in the future, since the proportion of children presenting with type 1 diabetes at a very young age is increasing continuously.

Acknowledgements This study was supported by the Medical Research Council, the Academy of Finland, the Novo-Nordisk Foundation, Finska Läkaresällskapet and the Liv and Hälsa Foundation. The Childhood Diabetes in Finland Study has been supported by grants from the Association of Finnish Life Insurance Companies, the Sigrid Jusélius Foundation, the National Institutes of Health (grant DK 37957) and the University of Helsinki, and the Study on Cardiovascular Risk Factors in Young Finns by the Academy of Finland, the Red Heart Operation Fund, the Social Insurance Institution of Finland, the National Board of Health, the Juho Vainio Foundation and the Yrjö Jahnsson Foundation. We thank S. Heikkilä and S. Anttila for their technical assistance. Some of these data were included in an Abstract presented at the 64th Scientific Sessions of the American Diabetes Association in 2004.

Duality of interest The authors declare that there is no duality of interest associated with this manuscript.

\section{References}

1. Pietropaolo M, Yu S, Libman IM et al (2005) Cytoplasmic islet cell antibodies remain valuable in defining risk of progression to type 1 diabetes in subjects with other islet autoantibodies. Pediatr Diabetes 5:184-192

2. Dittler J, Seidel D, Schenker M, Ziegler AG (1998) GADIA2combi determination as first-line screening for improved prediction of type 1 diabetes in relatives. Diabetes 47:592-597

3. Bingley PJ, Bonifacio E, Ziegler AG, Schatz DA, Atkinson MA, Eisenbarth GS (2001) Proposed guidelines on screening for risk of type 1 diabetes. Diabetes Care 24:398

4. LaGasse JM, Brantley MS, Leech NJ et al (2002) Successful prospective prediction of type 1 diabetes in schoolchildren through multiple defined autoantibodies: an 8-year follow-up of the Washington State Diabetes Prediction Study. Diabetes Care 25: 505-511

5. Schlosser M, Strebelow M, Wassmuth R et al (2002) The Karlsburg type 1 diabetes risk study of a normal schoolchild population: association of beta-cell autoantibodies and human leukocyte antigen-DQB1 alleles in antibody-positive individuals. J Clin Endocrinol Metab 87:2254-2261

6. Kulmala P, Savola K, Petersen JS et al (1998) Prediction of insulin-dependent diabetes mellitus in siblings of children with diabetes. A population-based study. The Childhood Diabetes in Finland Study Group. J Clin Invest 101:327-336

7. Åkerblom HK, Viikari J, Raitakari OT, Uhari M (1999) Cardiovascular risk in Young Finns Study: general outline and recent developments. Ann Med 31(Suppl 1):45-54

8. World Health Organization (1999) Definition, diagnosis and classification of diabetes mellitus and its complications: Report of a WHO consultation. WHO, Geneva (WHO/NCD/NCS 99.2)

9. Tuomilehto J, Virtala E, Karvonen M et al (1995) Increase in incidence of insulin-dependent diabetes mellitus among children in Finland. Int J Epidemiol 24:984-992

10. Savola K, Sabbah E, Kulmala P, Vähäsalo P, Ilonen J, Knip M (1998) Autoantibodies associated with type I diabetes mellitus persist after diagnosis in children. Diabetologia 41:1293-1297

11. Maclaren NK, Lan MS, Schatz D, Malone J, Notkins AL, Krischer J (2003) Multiple autoantibodies as predictors of type 1 diabetes in a general population. Diabetologia 46:873-874

12. Veijola R, Reijonen H, Vähäsalo P et al (1996) HLA-DQB1defined genetic susceptibility, beta cell autoimmunity, and metabolic characteristics in familial and nonfamilial insulin-dependent diabetes mellitus. Childhood Diabetes in Finland (DiMe) Study Group. J Clin Invest 98:2489-2495 